\title{
Changing-Look AGNs or Short-Lived Radio Sources?
}

\author{
Aleksandra Wołowska ${ }^{1 *}$, Magdalena Kunert-Bajraszewska ${ }^{1}$, Kunal Mooley $^{2}$ and \\ Gregg Hallinan ${ }^{3}$ \\ ${ }^{1}$ Toruń Centre for Astronomy, Faculty of Physics, Astronomy and Informatics, Nicolaus Copernicus University, Toruń, Poland, \\ ${ }^{2}$ Centre for Astrophysical Surveys, University of Oxford, Oxford, United Kingdom, ${ }^{3}$ Cahill Center for Astronomy, California \\ Institute of Technology, Pasadena, CA, United States
}

OPEN ACCESS

Edited by:

Paola Marziani,

Osservatorio Astronomico di Padova (INAF), Italy

Reviewed by: Brian Punsly,

International Center for Relativistic Astrophysics, Italy Nicolae Sebastian Zamfir, University of Wisconsin-Stevens Point,

United States

${ }^{*}$ Correspondence: Aleksandra Wołowska ola@astro.umk.pl

Specialty section:

This article was submitted to

Milky Way and Galaxies,

a section of the journal

Frontiers in Astronomy and Space

Sciences

Received: 31 August 2017 Accepted: 25 October 2017 Published: 17 November 2017

Citation:

Wołowska A, Kunert-Bajraszewska M,

Mooley K and Hallinan G (2017) Changing-Look AGNs or Short-Lived

Radio Sources?

Front. Astron. Space Sci. 4:38. doi: 10.3389/fspas.2017.00038
The evolution of extragalactic radio sources has been a fundamental problem in the study of active galactic nuclei for many years. A standard evolutionary model has been created based on observations of a wide range of radio sources. In the general scenario of the evolution, the younger and smaller Gigahertz-Peaked Spectrum (GPS) and Compact Steep Spectrum (CSS) sources become large-scale FRI and FRIl objects. However, a growing number of observations of low power radio sources suggests that the model cannot explain all their properties and there are still some aspects of the evolutionary path that remain unclear. There are indications, that some sources may be short-lived objects on timescales of $10^{4}-10^{5}$ years. Those objects represent a new population of active galaxies. Here, we present the discovery of several radio transient sources on timescales of 5-20 yrs, largely associated with renewed AGN (Active Galactic Nucleus) activity. These changing-look AGNs possibly represent behavior typical for many active galaxies.

Keywords: galaxies, active-galaxies, evolution, quasars, recurrent-activity

\section{INTRODUCTION}

The radio emission of extragalactic sources is usually explained by the existence of strong jets emitting non-thermal synchrotron radiation. However, AGNs with powerful jets represent only a small fraction of the entire population (Kellerman et al., 1989). The vast majority of sources are much less powerful in radio domain or even defined as radio-quiet. But even then they still emit radio waves at very small fluxes, compared to radio-loud AGNs. The relatively small amount of radio emission in some AGNs may be due the presence of less powerful jets (Ulvestad et al., 2005). This in turn connects the phenomena with the accretion process which can be radiatively inefficient in low radio luminosity AGNs (Merloni et al., 2003; Best and Heckman, 2012).

In the general scenario of the evolution of powerful radio-loud AGNs (Fanti et al., 1995), the younger and smaller Gigahertz-Peaked Spectrum (GPS) and Compact Steep Spectrum (CSS) sources become high luminosity large-scale FR II objects (Fanaroff and Riley, 1974; Figure 1). It seems reasonable to suspect that the compact AGNs with lower radio luminosity could be the progenitors of less luminous FR IIs and FR Is. However, the growing number of observations of low power radio sources and results of their analysis indicates that in their case the evolution can be more complicated or even halted at parsec scale. In order for the radio source to become a large-scale FR II or FR I object, the active phase needs to last longer than $10^{4}-10^{5}$ years. The shorter active phase, which is probably caused by the low accretion rate, will result in poorly developed, sometimes disrupted, compact radio morphology. Some AGNs may undergo numerous short phases during their lifetime (Reynolds and Begelman, 1997; Czerny et al., 2009; KunertBajraszewska et al., 2010). In this sense a "young" GPS or CSS source means ongoing episode of the 


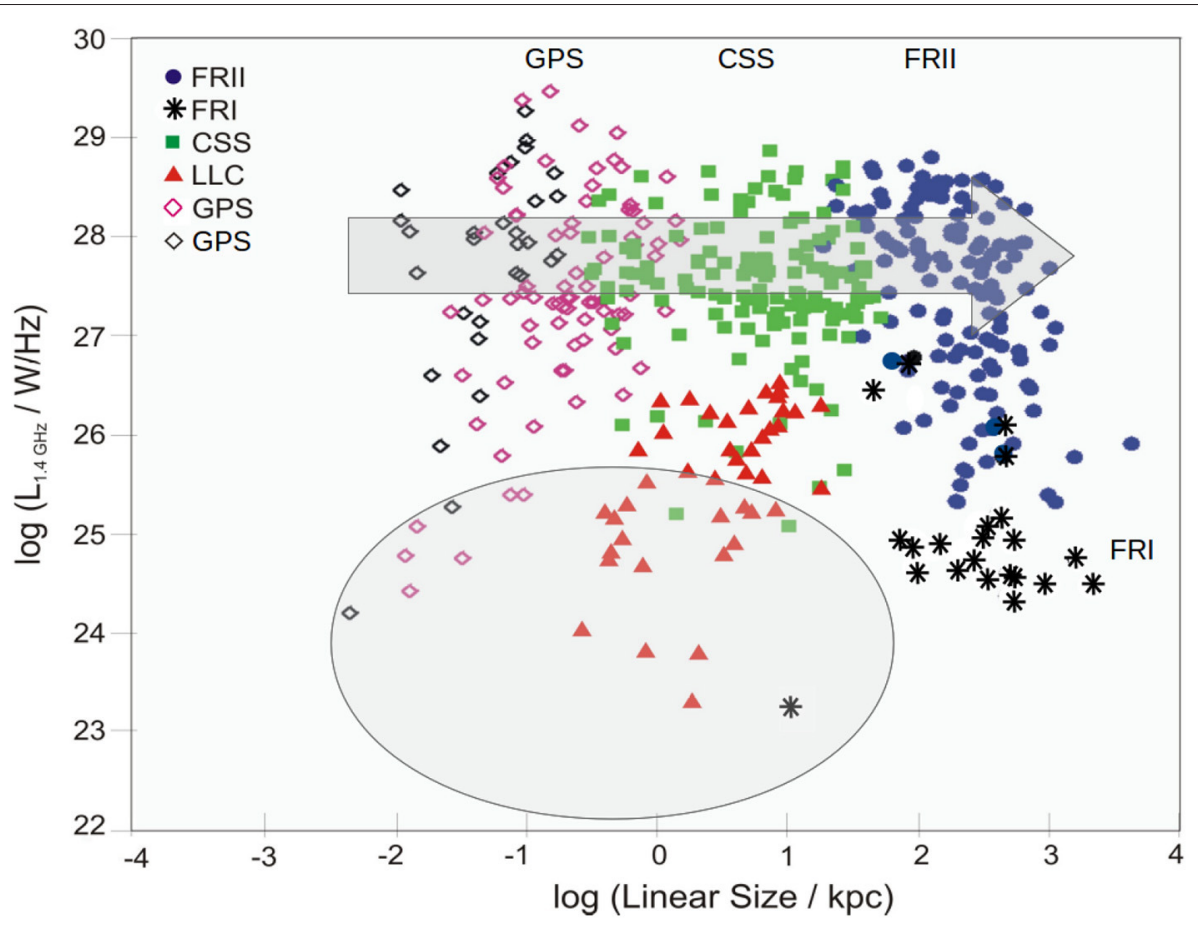

FIGURE 1 | Radio power vs. linear size evolutionary scheme for radio-loud AGN (Kunert-Bajraszewska, 2016, Copyright Wiley-VCH Verlag GmbH \& Co. KGaA. Reproduced with permission). Squares represent CSS sources (Laing et al., 1983; Fanti et al., 2001; Marecki et al., 2003). Diamonds indicate GPS objects (Labiano et al., 2007). Stars indicate FRI and FRII objects (Laing et al., 1983). Red triangles represent low luminosity compact (LLC) sources previously studied by (Kunert-Bajraszewska et al., 2010, 2014; Kunert-Bajraszewska and Labiano, 2014). The gray arrow indicates the main evolutionary trend of radio-loud AGNs. The oval indicates the boundaries of the area in which the new radio transient sources are located and where, as we expect, the whole population of short-lived objects can be found.

accretion disk outburst. This indicates the temporary existence of many weak CSS and GPS sources with compact or slightly resolved radio morphologies (core-jet) similar to that observed in radio-quiet objects (Giroletti and Panessa, 2009; Sadler et al., 2014). They are called short-lived radio objects on timescales $10^{4}-10^{5}$ years. Interestingly, the enhancement and cessation of the accretion process is one of the postulated explanation for the origin of changing-look behavior in the so-called "changing look AGNs" (Elitzur et al., 2014; Schawinski et al., 2015). Such objects are rare and their first discoveries were made based on the X-ray variability. However, Koay et al. (2016) recently reported the changing-look behavior in radio domain in one of such sources. This, in our opinion, links the discussion about the changing-look AGNs with the subject of short term radio activity.

To this day, only a handful of radio-loud short-lived AGN candidates have been found and studied (Kunert-Bajraszewska et al., 2010, 2014). However, the analysis of the radio, optical and X-ray proper- ties of a sample of low-luminosity compact (LLC) radio sources carried out by our group over the last few years (Figure 1) suggests that a much larger population of such radio sources exist, and need to be explored. The first unbiased study of these sources has been recently carried out with the Jansky VLA. The multi-year Caltech-NRAO Stripe 82 Survey (CNSS), revealed twelve transient sources on timescales of 5-20 yrs, largely associated with renewed AGN activity. The rates of such AGN possibly imply episodes of enhanced accretion and jet activity occurring once every 40,000 years in these galaxies. The whole CNSS consist of five epochs of observations over the entire $\sim 270 \mathrm{deg}^{2}$ of Stripe 82 and they will be published soon. The results from an initial pilot survey of a $\sim 50 \mathrm{deg}^{2}$ of Stripe 82 has been already published by Mooley et al. (2016).

\section{NEW RADIO TRANSIENTS}

The CNSS is a dedicated radio transient survey carried out with the Jansky VLA between December 2012 and May 2015. It was designed for systematically exploring the radio sky for slow transient phenomena on timescales between one day and several years. Observations of the $270 \mathrm{deg}^{2}$ of the SDSS Stripe 82 region were carried out over 5 epochs at $3 \mathrm{GHz}$ and with a uniform rms noise of $80 \mu \mathrm{Jy}$ per epoch. The CNSS survey has facilitated an unbiased study of short-lived sources for the first time.

In this survey, 50 radio transients were discovered, among which are AGN, candidates of tidal disruption events and stellar explosions, flare stars, and active binary star systems (Mooley et al., 2016). Although the majority of radio flaring phenomena in AGN are due to shocks propagating down the jets (e.g., Marscher and Gear, 1985), properties of some AGNs discovered as radio transients are distinct from this flaring population.

This distinct population comprises AGNs that were not detected in radio in any previous survey of Stripe 82. They 

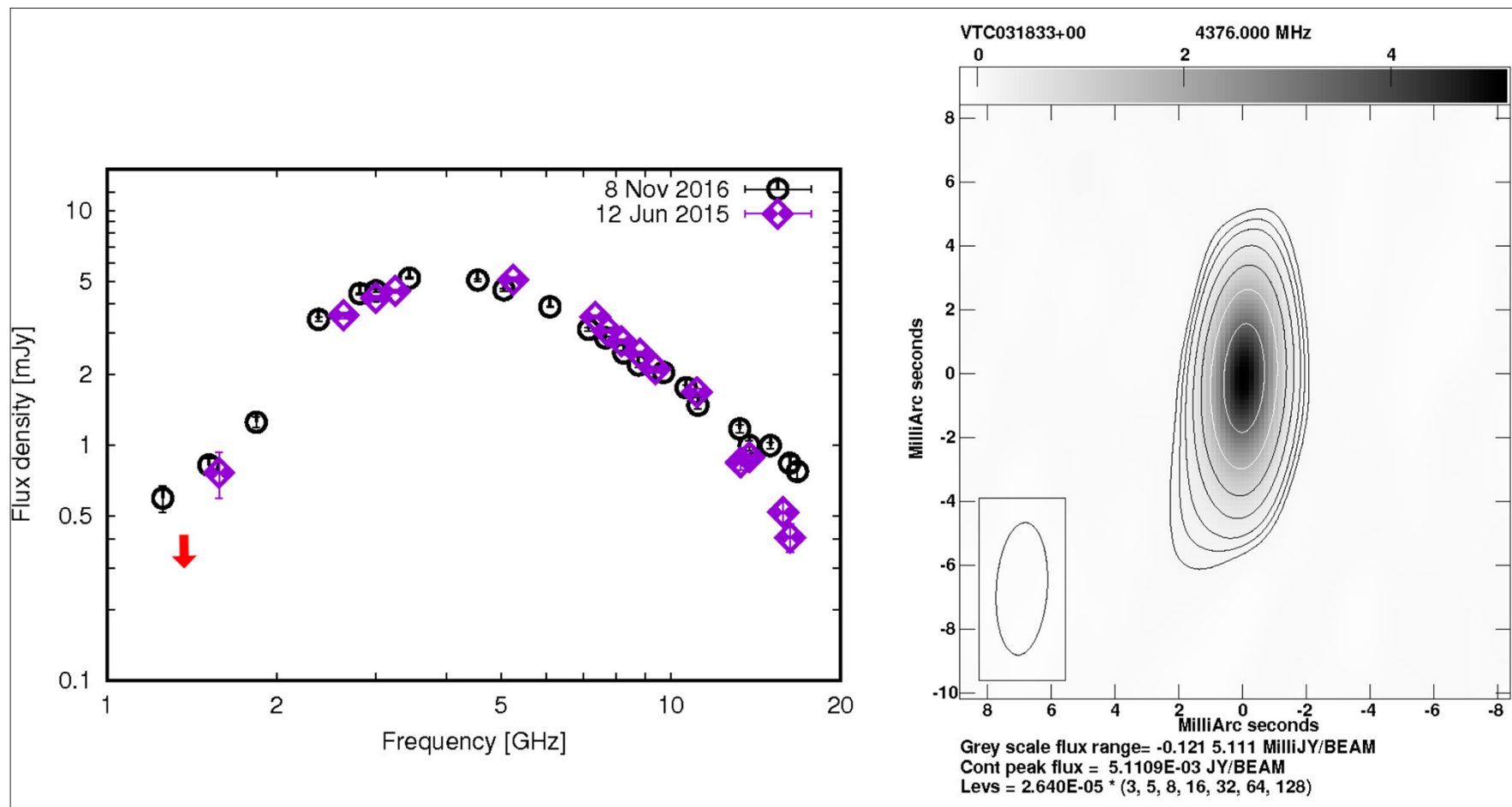

FIGURE 2 | The 1-20 GHz VLA spectrum and 4.5 GHz VLBA image of VTC031833+00. The sensitivity limit of the FIRST survey (1.4 GHz, mean epoch 1999.2) is shown as a red arrow on the spectrum plot.

have been radio-quiet sources so far. After the outburst, the new radio sources are characterised by convex radio spectra peaking at a few $\mathrm{GHz}$, which is typical for young AGNsGPS sources. From our set of optical observations, we have determined the spectral types of these objects to be the second kind. In the type-II objects the central nucleus is obscured by a molecular-dusty torus, which means that the direction of view is close to the plane of the disk. This significantly reduces the possibility of the Doppler enhancing of the jet flux fluctuations which can mimic the birth of new jet activity.

One such AGN, the type II quasar VTC233002-00 at redshift $z=1.65$ has been recently reported by Mooley et al. (2016). VTC233002-00 was detected at $\sim 5.5 \mathrm{mJy}$ at $3 \mathrm{GHz}$ in the CNSS, while the $3 \sigma$ upper limit at $1.4 \mathrm{GHz}$ is $0.4 \mathrm{mJy}$ (mean epoch 1999.2). The order-of-magnitude increase in flux density could be indicative of an enhanced accretion phenomenon leading to the production of a new jet. A comparison between the radio and optical flux densities indicates that this is a radio loud quasar. However, the value of the radio-loudness parameter $(\log R)$ calculated before and after the outburst changed from $\log \mathrm{R}<1$ to $\log \mathrm{R}=2.1$, respectively. This indicates that VTC23300200 has changed its status (look) from radio-quiet to radio-loud source ${ }^{1}$.

${ }^{1}$ We adopted radio-loudness definition from Kimball et al. (2011): $\log \mathrm{R}=$ $\left(\mathrm{M}_{\text {radio }}-\mathrm{M}_{\mathrm{i}}\right) /-2.5$, where $\mathrm{M}_{\text {radio }}$ is a K-corrected radio absolute magnitude and $\mathrm{M}_{\mathrm{i}}$ is a Galactic reddening corrected and K-corrected i-band absolute magnitude. Source is considered to be radio-loud if $\log \mathrm{R}>1$.
Finally, 12 such objects have been identified and we have undertaken a multi-frequency follow up campaign for those transient objects. New radio and X-ray observations and their analysis will be published soon. The latest preliminary results of our follow up VLA and VLBA observations of another of these objects are presented in Figure 2. VTC031833+00 has been discovered in 2015 in the CNSS survey and the Jansky VLA observations at 1-20 GHz carried by us in 2016 confirmed it is a GPS source peaking at $5 \mathrm{GHz}$ with a flux density of $5.1 \mathrm{mJy}$. The peak seems to slightly move towards lower frequency, when comparing the spectrum from June 2015 and the 2016 spectrum. That process could be due to propagating of the jet and its interaction with the circumnuclear material in the host galaxy. The $4.5 \mathrm{GHz}$ VLBA image of VTC031833+00 shows slightly resolved radio structure, with a flux density of $5.1 \mathrm{mJy}$, consistent with published VLA results. This radio morphology is similar to those of the radio-quiet AGNs probably indicative of weak jets that are not able to develop large-scale structure (Ulvestad et al., 2005).

\section{SUMMARY}

Our sample represents a special class of radio-loud AGNs that harbor jets switched on within the past few decades. These are, thus, the youngest radio sources that can provide information about the accretion state of supermassive black holes shortly after the onset of the jet formation. Furthermore, 
studies of interactions between the young radio source and interstellar medium can provide information about the energy that is deposited into the ISM by the expanding radio source. This is important to our understanding of the feedback process. Taking this into account, we have undertaken a multifrequency follow up campaign for the discovered transient objects. The detailed studies of their morphology and the jet evolution as well as the discussion about the postulated short term activity of AGNs will be published in forthcoming papers.

\section{REFERENCES}

Best, P. N., and Heckman, T. M. (2012). On the fundamental dichotomy in the local radio-AGN population: accretion, evolution and host galaxy properties. Month. Notices R. Astron. Soc. 421, 1569-1582. doi: 10.1111/j.1365-2966.2012.20414.x

Czerny, B., Siemiginowska, A., Janiuk, A., Nikiel-Wroczyński, B., and Stawarz, Ł. (2009). Accretion disk model of short-timescale intermittent activity in young radio Sources. Astrophys. J. 698, 840-851. doi: 10.1088/0004-637X/698/1/840

Elitzur, M., Ho, L. C., and Trump, J. R. (2014). Evolution of broad-line emission from active galactic nuclei. Month. Notices R. Astron. Soc. 438, 3340-3351. doi: $10.1093 / \mathrm{mnras} / \mathrm{stt} 2445$

Fanaroff, B. L., and Riley, J. M. (1974). The morphology of extragalactic radio sources of high and low luminosity. Month. Notices R. Astron. Soc. 167, 31P-36P. doi: 10.1093/mnras/167.1.31P

Fanti, C., Fanti, R., Dallacasa, D., Schilizzi, R. T., Spencer, R. E., and Stanghellini, C. (1995). Are compact steep-spectrum sources young? Astron. Astrophys. 302, 317-326.

Fanti, C., Pozzi, F., Dallacasa, D., Fanti, R., Gregorini, L., Stanghellini, C., et al. (2001). Multi-frequency VLA observations of a new sample of CSS/GPS radio sources. Astron. Astrophys. 369, 380-420. doi: 10.1051/0004-6361:20010051

Giroletti, M., and Panessa, F. (2009). The faintest seyfert radio cores revealed by VLBI. Astrophys. J. Lett. 706, L260-L264. doi: 10.1088/0004-637X/706/2/L260

Kellerman, K. I., Sramek, R., Schmidt, M., Shaffer, D. B., and Green, R. (1989). VLA Observations of objects in the palomar bright quasar survey. Astron. J. 98, 1195-1207.

Kimball, A. E., Ivezić, Ž., Wiita, P. J., and Schneider, D. P. (2011). Correlations of quasar optical spectra with radio morphology. Astron. J. 141:182. doi: 10.1088/0004-6256/141/6/182

Koay, J. Y., Vestergaard, M., Bignall, H. E., Reynolds, C., and Peterson, B. M. (2016). Parsec-scale radio morphology and variability of a changing-look AGN: the case of Mrk 590. Month. Notices R. Astron. Soc. 460, 304-316. doi: $10.1093 / \mathrm{mnras} / \mathrm{stw} 975$

Kunert-Bajraszewska, M. (2016). Dichotomy in the population of young AGN: optical, radio, and X-ray properties. Astron. Nachr. 337, 27-30. doi: 10.1002/asna.201512259

Kunert-Bajraszewska, M., Gawroński, M. P., Labiano, A., and Siemiginowska, A. (2010). A survey of low-luminosity compact sources and its implication for the evolution of radio-loud active galactic nuclei - I. Radio data. Month. Notices $R$. Astron. Soc. 408, 2261-2278. doi: 10.1111/j.1365-2966.2010.17271.x

Kunert-Bajraszewska, M., and Labiano, A. (2014). A survey of low-luminosity compact sources and its implication for the evolution of radio-loud active galactic nuclei - II. Optical analysis. Month. Notices R. Astron. Soc. 408, 2279-2289. doi: 10.1111/j.1365-2966.2010.17300.x

Kunert-Bajraszewska, M., Labiano, A., Siemiginowska, A., and Guainazzi, M. (2014). First X-ray observations of low-power compact steep spectrum sources. Month. Notices R. Astron. Soc. 437, 3063-3071. doi: 10.1093/mnras/stt1978

\section{AUTHOR CONTRIBUTIONS}

All authors participated in acquisition, analysis and interpretation of data as well as the preparation of the manuscript.

\section{ACKNOWLEDGMENTS}

This is the contribution to the proceedings of the conference "Quasars at all cosmic epochs," held in Padua (Italy) in April 2017.

Labiano, A., Barthel, P. D., O’Dea, C. P., de Vries, W. H., Prez, I., and Baum, S. A. (2007). GPS radio sources: new optical observations and an updated master list. Astron. Astrophys. 463, 97-104. doi: 10.1051/0004-6361:200 66183

Laing, R. A., Riley, J. M., and Longair, M. S. (1983). Bright radio sources at 178 $\mathrm{MHz}$ - Flux densities, optical identifications and the cosmological evolution of powerful radio galaxies. Month. Notices R. Astron. Soc. 204, 151-187. doi: $10.1093 / \mathrm{mnras} / 204.1 .151$

Marecki, A., Spencer, R. E., and Kunert, M. (2003). Location of Weak CSS Sources on the Evolutionary path of radio-loud AGN. Astron. Soc. Aust. 20, 46-49. doi: 10.1071/AS02051

Marscher, A. P., and Gear, W. K. (1985). Models for high-frequency radio outbursts in extragalactic sources, with application to the early 1983 millimeter-to-infrared flare of 3C 273. Astron. J. 298, 114-127. doi: 10.1086/ 163592

Merloni, A., Heinz, S., and di Matteo, T. (2003). A Fundamental Plane of black hole activity. Month. Notices R. Astron. Soc. 345, 1057-1076. doi: 10.1046/j.1365-2966.2003.07017.x

Mooley, K. P., Hallinan, G., Bourke, S., Horesh, A., Myers, S. T., Frail, D. A., et al. (2016). The Caltech-NRAO Stripe 82 Survey (CNSS). I. The pilot radio transient survey in $50 \mathrm{deg}^{2}$. Astrophys. J. 818:105. doi: 10.3847/0004-637X/ $818 / 2 / 105$

Reynolds, C. S., and Begelman, M. C. (1997). Intermittant radio galaxies and source statistics. Astrophys. J. 487, L135-L138.

Sadler, E. M., Ekers, R. D., Mahony, E. K., Mauch, T., and Murphy, T. (2014). The local radio-galaxy population at $20 \mathrm{GHz}$. Month. Notices R. Astron. Soc. 438, 796-824. doi: 10.1093/mnras/stt2239

Schawinski, K., Koss, M., Berney, S., and Sartori, L. F. (2015). Active galactic nuclei flicker: an observational estimate of the duration of black hole growth phases of $10^{5} \mathrm{yr}$. Month. Notices R. Astron. Soc. 451, 2517-2523. doi: $10.1093 / \mathrm{mnras} / \mathrm{stv} 1136$

Ulvestad, J. S., Antonucci, R. R. J., and Barvainis, R. (2005). VLBA imaging of central engines in radio-quiet quasars. Astrophys. J. 621, 123-129. doi: 10.1086/ 427426

Conflict of Interest Statement: The authors declare that the research was conducted in the absence of any commercial or financial relationships that could be construed as a potential conflict of interest.

Copyright (C) 2017 Wołowska, Kunert-Bajraszewska, Mooley and Hallinan. This is an open-access article distributed under the terms of the Creative Commons Attribution License (CC BY). The use, distribution or reproduction in other forums is permitted, provided the original author(s) or licensor are credited and that the original publication in this journal is cited, in accordance with accepted academic practice. No use, distribution or reproduction is permitted which does not comply with these terms. 